\title{
Modifying the strength and strain concentration profile within collagen scaffolds using customizable arrays of poly-lactic acid fibers
}

\author{
Laura C. Mozdzen ${ }^{\mathrm{a}}$, Alan Vucetic ${ }^{\mathrm{a}}$, Brendan A.C. Harley ${ }^{\mathrm{a}, \mathrm{b}}$ \\ ${ }^{a}$ Department of Chemical and Biomolecular Engineering \\ ${ }^{\mathrm{b}}$ Carl R. Woese Institute for Genomic Biology \\ University of Illinois at Urbana-Champaign \\ Urbana, IL 61801 \\ United States
}

\section{Corresponding Author:}

B.A.C. Harley

Dept. of Chemical and Biomolecular Engineering

Carl R. Woese Institute for Genomic Biology

University of Illinois at Urbana-Champaign

110 Roger Adams Laboratory

600 S. Mathews Ave.

Urbana, IL 61801

Phone: (217) 244-7112

Fax: (217) 333-5052

email: bharley@illinois.edu 


\begin{abstract}
The tendon-to-bone junction is a highly specialized tissue which dissipates stress concentrations between mechanically dissimilar tendon and bone. Upon injury, the local heterogeneities across this insertion are not regenerated, leading to poor functional outcomes such as formation of scar tissue at the insertion and re-failure rates exceeding 90\%. Although current tissue engineering methods are moving towards the development of spatially-graded biomaterials to begin to address these injuries, significant opportunities remain to engineer the often complex local mechanical behavior of such biomaterials to enhance their bioactivity. Here, we describe the use of three-dimensional printing techniques to create customizable arrays of poly-lactic acid (PLA) fibers that can be incorporated into a collagen scaffold under development for tendon bone junction repair. Notably, we use additive manufacturing concepts to generate arrays of spatiallygraded fibers from biodegradable PLA that are incorporated into collagen scaffolds to create a collagen-PLA composite. We demonstrate the ability to tune the mechanical performance of the fiber-scaffold composite at the bulk scale. We also demonstrate the incorporation of spatiallyheterogeneous fiber designs to establish non-uniform local mechanical performance of the composite biomaterial under tensile load, a critical element in the design of multi-compartment biomaterials for tendon-to-bone regeneration applications. Together, this work highlights the capacity to use multi-scale composite biomaterials to control local and bulk mechanical properties, and provides key insights into design elements under consideration for mechanically competent, multi-tissue regeneration platforms.
\end{abstract}

Keywords: collagen; scaffold; fiber-reinforced; composite; spatially-graded; 3D printing 


\section{1: Introduction}

The tendon-to-bone junction (TBJ) is a highly specialized transition region which connects elastic tendon to stiff, mineralized bone for the purpose of movement through the translation of high tensile forces. The local gradients of matrix proteins and structural organization across the transitional region serves to both transmit loads between tendon and bone, but also dissipate stress concentrations which can form at the interface between mechanically mismatched materials (Genin et al., 2009; Killian et al., 2012; Li et al., 2009). However, injuries to the TBJ such as those often seen in rotator cuff tears and other orthopedic insertion injuries require more than 4.5 million physician visits and more than 250,000 surgeries each year in the US alone (Yamaguchi et al., 2006). Despite the high prevalence of this injury, surgical outcomes are poor and re-failure rates are quite high $(>90 \%$ in elderly populations) because the insertion is not regenerated during the healing process (Genin et al., 2009). This poor functional outcome motivates the development of tissue engineering solutions to improve the regenerative healing capacity of the osteotendinous enthesis.

Emerging efforts have begun to shift focus from homogenous biomaterials for the purpose of regenerating a single tissue (Caliari et al., 2011; Ferreira et al., 2012; Galatz et al., 2006; Kanungo et al., 2008; Levingstone et al., 2014; Mathew et al., 2012) to multi-compartment and spatially graded biomaterials for the express purpose of repairing more complex tissues such as the tendon-to-bone junction (Caliari et al., 2015a; Harley et al., 2010; Lipner et al., 2014; Qu et al., 2013; Smith et al., 2016; Weisgerber et al., 2015; Weisgerber et al., 2013b). Efforts in our lab have recently described two new variants of collagen scaffolds under development for osteotendinous repair applications. The first is a collagen scaffold that contains distinct 
mineralized and non-mineralized collagen compartments joined by a continuous interface that utilize changes in local biophysical properties to induce divergent osseous and tendinous differentiation of human MSCs (Caliari and Harley, 2014; Caliari et al., 2015b). The second platform technology was targeted to address the poor mechanical performance of the porous collagen scaffold, incorporating ABS fibers as reinforcing elements to form a collagen-ABS composite. Here, the composite displayed tunable mechanical properties that were significantly greater than that of the native scaffold, providing an avenue to create a monolithic scaffold with enhanced mechanical properties (Mozdzen et al., 2016). Further, we showed that the ABS fiber could be covalently functionalized with biomolecules (e.g., PDGF) to enhance the bioactivity of cells encapsulated within the composite (Mozdzen et al., 2016). However, while straightforward to fabricate using commercially available three-dimensional printers, a range of translational concerns associated with biocompatibility and degradation limited the long-term use of nongrowth factor functionalized ABS (Mozdzen et al., 2016).

This work expands upon these previous biomaterial designs to demonstrate a fiber-reinforced collagen biomaterial that: (1) incorporates biocompatible and biodegradable PLA fibers; and (2) uses arrays of spatially-graded fibers in order to locally manipulate the mechanical performance of the composite biomaterial. Here we first describe the incorporation of arrays of PLA fibers fabricated via three-dimensional printing into a monolithic collagen-GAG (CG) scaffold in order to enhance the overall tensile properties of the composite. We subsequently describe the use of spatially graded fibers with either discontinuous or gradient profiles in order to tune local tensile behavior of the scaffold-composites in order to generate regionally stiffer and more elastic zone conducive to tendon-to-bone repair applications where tendon and bone can have up to two order 
of magnitude difference in mechanical properties (Thomopoulos et al., 2003). As heterogeneous materials can be difficult to characterize via traditional bulk characterization techniques due to an inability to locally monitor mechanical behavior (Stops et al., 2008), we employ digital image correlation methods to locally map strain profiles across these scaffolds in order to more accurately resolve the mechanical stimuli that would locally impact cells seeded within these biomaterial composites.

\section{2: Materials and Methods}

\subsection{Preparation of collagen-GAG $(C G)$ suspension}

A CG suspension was prepared from type I collagen $(1.0 \% \mathrm{w} / \mathrm{v})$ isolated from bovine Achilles tendon and chondroitin sulfate $(0.1 \% \mathrm{w} / \mathrm{v})$ derived from shark cartilage in $0.05 \mathrm{M}$ acetic acid (Sigma-Aldrich, St. Louis, MO) (Yannas et al., 1989). The suspension was homogenized at $4{ }^{\circ} \mathrm{C}$ to prevent gelatinization during mixing and was degassed before use (Yannas et al., 1989).

\subsection{Fabrication of polylactic acid fiber arrays via $3 D$ printing}

Each PLA fiber array consisted of 9 parallel $1 \mathrm{~mm}$ diameter PLA fibers $(20 \mathrm{~mm}$ wide $\times 76 \mathrm{~mm}$ long) fixed together at either end with PLA end blocks (20 mm $\times 4 \mathrm{~mm} \times 3 \mathrm{~mm}$ thick). All constructs were printed using a MakerBot Replicator 2X (MakerBot Industries, Brooklyn, NY) at standard quality settings (10\% infill, 2 shells, $200 \mu \mathrm{m}$ layered height) and an extruder speed of $25 \mathrm{~mm} / \mathrm{s}$. To ensure structural fidelity of the fiber arrays, PLA fibers were co-printed with polystyrene supports and rafts (base layer) using dual nozzles (polystyrene: $250{ }^{\circ} \mathrm{C}$; PLA: 210 ${ }^{\circ} \mathrm{C}$ ) onto a temperature controlled build-plate $\left(55^{\circ} \mathrm{C}\right)$. The polystyrene supports were 
subsequently dissolved in D-Limonene (GreenTerpene, Miami, FL), leaving behind the final PLA fiber array. After polystyrene dissolution, the PLA fiber arrays were washed multiple times with deionized water ( $3 \times 20$ minutes), and then dried before use.

A homologous series of reinforcing arrays were created to explore the effect of local fiber geometry on mechanical performance. One set of fiber arrays was comprised of uniform fiber architecture along its length; these arrays were created using sinusoidal fiber architectures with either a $1 \mathrm{~mm}$ or $2 \mathrm{~mm}$ sinusoidal amplitude. This first group (continuous) displayed the same geometries reported in our previous work using ABS fibers (Mozdzen et al., 2016). Here, fiber arrays were either linear (straight fiber array: Straight) or were sinusoidal with a set period (11.3 $\mathrm{mm}$ ) and one of two amplitudes (1 $\mathrm{mm}$ sinusoidal fiber array: $1 \mathrm{~mm} \mathrm{Amp;} 2 \mathrm{~mm}$ sinusoidal fiber

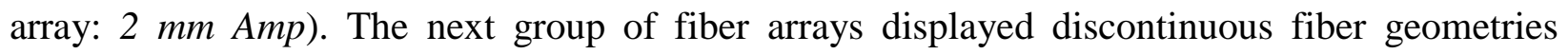
(discontinuous), with the length of the fiber divided into two regions, with a straight fiber in one half of the array joined to either a $1 \mathrm{~mm}$ or a $2 \mathrm{~mm}$ sinusoidal fiber on the other side (discontinuous: $1 \mathrm{~mm}$ Amp, $2 \mathrm{~mm} \mathrm{Amp).} \mathrm{The} \mathrm{final} \mathrm{group} \mathrm{of} \mathrm{fiber} \mathrm{arrays} \mathrm{also} \mathrm{displayed}$ discontinuous geometries, but contained a gradual transition across its length (straight; $1 / 4$ amplitude, $1 / 2$ amplitude, full amplitude) using both the $1 \mathrm{~mm}$ and $2 \mathrm{~mm}$ sinusoidal fiber array (gradient: $1 \mathrm{~mm}$ Amp, $2 \mathrm{~mm} \mathrm{Amp)} \mathrm{(Figure} \mathrm{1).} \mathrm{We} \mathrm{have} \mathrm{previously} \mathrm{published} \mathrm{detailed} \mathrm{methods}$ regarding the incorporation of (acrylonitrile butadiene styrene, ABS) polymer fibers into CG scaffolds that includes SEM images of fibers within the scaffold and failure modes under supraphysiological strain (Mozdzen et al., 2016).

\subsection{Fabrication of PLA fiber reinforced CG scaffolds via lyophilization}


PLA fiber arrays were subsequently incorporated into CG scaffolds during lyophilization as previously described (Mozdzen et al., 2016). Briefly, after printing, PLA fiber arrays were placed into aluminum molds $7.6 \mathrm{~cm}$ (long) $\times 2 \mathrm{~cm}$ (wide) and $8.7 \mathrm{~mL}$ of $\mathrm{CG}$ suspension was added to submerge the fiber array. The final PLA-CG composite was fabricated via a previously described lyophilization protocol (-40 ${ }^{\circ} \mathrm{C}, 200$ mTorr) (Mozdzen et al., 2016; O'Brien et al., 2004), leaving behind the porous CG scaffold impregnated with PLA fibers. Scaffold composites $(7.6 \mathrm{~cm} \times 2$ $\mathrm{cm} \times 0.5 \mathrm{~cm}$ ) were stored under desiccation until use.

\subsection{Sample preparation for mechanical testing and digital image correlation (DIC)}

Collagen-PLA composites were speckle patterned using an airbrush (nozzle: $0.3 \mathrm{~mm}$; gravity feed; GotHobby, South El Monte, CA) filled with waterproof India ink (Blick Art Materials, Galesburg, Illinois) attached to house air before use (Lionello et al., 2014; Myers et al., 2010).

\subsection{Bulk mechanical characterization}

Fiber-scaffold composites (20 mm width, $5 \mathrm{~mm}$ thickness, $60 \mathrm{~mm}$ gauge length) underwent mechanical tensile testing in an Instron 5943 Mechanical Testing System with a $100 \mathrm{~N}$ electromechanical load frame. All tests were performed on composites in the dry (non-hydrated state) to facilitate Digital Image Correlation analysis of local strain profiles. Samples were held in place at their end-blocks with pneumatic grips (60 psi). Composites were strained at a rate of 6 $\mathrm{mm} / \mathrm{min}(0.167 \%$ strain/s) until failure, with full-sample images captured using a high definition digital camera (Canon EOS E5) with a $100 \mathrm{~mm}$ lens (Canon $100 \mathrm{~mm}$ macro lens) and a time lapse remote (Canon Timer Remote Controller TC-80N3) at a rate of 1 image every 2 seconds. Elastic modulus was calculated from the slope of the linear elastic region of the stress-strain 
curve (Harley et al., 2007; Maganaris and Paul, 1999), peak stress was taken as the maximum stress the sample experienced before failure, toughness was calculated as the area under the stress-strain curve after failure (Chandrashekar et al., 2012), and failure mode was recorded by taking the final image captured after failure.

\subsection{Local mechanical characterization}

Digital Image Correlation was used to resolve spatially-graded strain profiles within the collagen-PLA composites. Images stacks taken during tensile testing were analyzed using the Improved Digital Image Correlation code (Improved Digital Image Correlation v4 - C 2013, 2014, 2015 by Elizabeth Jones) (Jones, 2013) to calculate local strain profiles across each specimen. Reduced images were correlated first, with individual correlation settings optimized for each sample (typical settings: image reduction factor: 7, subset size: 35; threshold: 0.3 ; search zone: 4, grid step size: 15). Reduced correlations were iterated up to 6 times due to high strain. Full images were then correlated using the reduced data (typical settings: subset size: 21; threshold 0.3; search zone: 3; grid step size: 23). Finally, correlated data was smoothed and missing data (applicable at higher strains not included in the visual representations) was interpolated (Gaussian distribution of weights; kernel size: 11; number of smoothing passes: 3; maximum size of contiguous non-correlated points to smooth over: 35) with local strain profiles calculated using a cubic (16-node) strain algorithm (Szabo and Babuska, 1991; Zienkiewicz, 1977). The "visualize data" feature of the Improved Digital Image Correlation code was used to generate contour plots and line scans of strain across each specimen (Jones, 2013). This data was exported to excel to quantify maximum strain gradient and maximum local strain across each specimen. Strain gradients were calculated along the length of the sample using 9 line scans 
(evenly spaced across the width of the sample) of local strain, with the maximum slope taken as the maximum strain gradient (Abd Elhady, 2010; Marino and Vairo, 2014; Sano et al., 2013). Maximum local strain was taken as the highest local strain value across both the entire sample, as well as the middle $1 / 3$ of the sample, where fiber geometries either changed in a discrete (discontinuous) or gradual (gradient) manner.

\subsection{Statistics}

One-way analysis of variance (ANOVA) and two-way ANOVA (when two variables were changed in parallel) was performed on elastic modulus, peak stress, and toughness, and maximum strain gradient calculations $(n=6)$, followed by Tukey post-hoc tests. Significance was set at $p<0.05$. Error is reported as standard error of the mean unless otherwise noted.

\section{3: Results}

\subsection{Bulk mechanical characterization of PLA collagen composites}

The elastic moduli of the composites were found to significantly vary among each sample group. Consistent with previous findings using ABS fibers, the bulk elastic modulus of the composite increased significantly as the amplitude of the sinusoidal PLA fiber array decreased. The bulk elastic modulus also increased significantly with increasing spatial complexity of the fiber arrays $\left(E_{\text {gradient }}>E_{\text {discontinuous }}>E_{\text {continuous }}\right.$, Figure 2).

\subsection{Failure modes}

While it was possible to determine elastic moduli for all groups, the increased stiffness of PLA 
fibers resulted in some composites (some variants using either the $1 \mathrm{~mm}$ sinusoidal amplitude or the straight fiber) that did not fail in tension using the most robust load cell $(100 \mathrm{~N})$ available for our testing apparatus. As a result we subsequently examined failure properties of a library of all spatially-variable fiber geometries (continuous, discontinuous, gradient) using the most compliant $2 \mathrm{~mm}$ sinusoidal amplitude. Failure occurred randomly in the continuous samples, and occurred in the sinusoidal region of the discontinuous and gradient samples. Notably, while toughness of the PLA-collagen composite remains constant among the continuous, discontinuous, and gradient fiber geometries, overall peak stress was increased significantly in the gradient fiber geometry (Figure 3).

\subsection{Qualitative analysis of strain fields within PLA-collagen composites}

Subsequent analysis of local strain fields within the PLA-collagen composite were acquired for the range of continuous, discontinuous, and gradient fiber arrays (not straight fibers). Strain images were generated via digital image correlation for up to $3.7 \%$ applied (bulk) strain for visualization purposes. After images were correlated, local strain contour plots were compared to examine patterns in strain concentrations. Composites made up of arrays of continuous fibers show periodic areas of high strain, which correspond to the geometry of the sinusoidal fiber embedded in the scaffold. These areas of local strain were more pronounced within the $1 \mathrm{~mm}$ Amp sample. Composites made up of arrays of discontinuous fibers displayed areas of high periodic strain primarily in the half of the composite containing the sinusoidal fiber segment; again, areas of local strain appeared sharper in the $1 \mathrm{~mm}$ vs. $2 \mathrm{~mm}$ Amp group, where regions of high strain fields were more spread. Similar to the discontinuous sample groups, composites containing arrays of gradient fibers displayed high levels of local strain confined to the 
sinusoidal fiber region. Although there appears to be a gradual region of increasing strain, the local strain increased more sharply when the gradient transition reached the full amplitude of the fiber (Figure 4).

\subsection{Quantifying the distribution of local strain profiles across the PLA-collagen composite}

In order to better quantify the distribution of strain across the composites, a series of 9 line scans were taken along the length of each composite. Line scans were acquired in the direction of applied strain, with each scan evenly spaced in the direction perpendicular to strain. Scans were acquired at a series of applied bulk strains $(0,1.0,1.7,2.3,3.0,3.7 \%)$ in order to quantify the distribution of strain across the composite as well as to calculate maximum strain gradients for individual line scans (Figure 5).

The distribution of stain fields along the length of each specimen were quantified for the library of continuous, discontinuous, and gradient fiber reinforced PLA-collagen composites. Local strain profiles were first characterized for these specimens over a range of applied strains $(0,1.0$, 1.7, 2.3, 3.0, 3.7\%) (Figure 6). However, some samples (1 mm Amp gradient, $1 \mathrm{~mm} \mathrm{Amp}$ discontinuous) were unable to reach the highest levels of strain given limitation on the load cell of our system; in those cases, local strain maps were generated for all achievable bulk tensile strains. Here, increasing levels of local strain are seen with increasing applied bulk strain.

In order to specifically compare local strain profiles between all specimens, samples were compared at $2.3 \%$ applied (bulk) strain, the largest bulk strain all samples achieved without exceeding the load cell. All samples retained composite mechanical fidelity at $2.3 \%$ applied 
(bulk) strain. Here, results are shows as the mean local strain (dark lines) along with the standard deviation (light gray lines) across a representative sample for each group (Figure 7).

Together, these analyses show two important results. With increasing bulk strain, the continuous samples both displayed increasingly high areas of local strain. Local strain was more evenly spread along the $2 \mathrm{~mm}$ sample, with less sharp periodicity, while in the $1 \mathrm{~mm}$ sample local strain was more sharply periodic. In the discontinuous and gradient sample groups, high areas of periodic strain appeared primarily on one half, which corresponds to the sinusoidal part of the discontinuous or gradient fiber composite. Further, local areas of strain appeared sharper in the 1 mm Amp group compared to the $2 \mathrm{~mm} \mathrm{Amp} \mathrm{group,} \mathrm{which} \mathrm{were} \mathrm{spread} \mathrm{out} \mathrm{over} \mathrm{a} \mathrm{greater} \mathrm{distance.}$ However, when compared to the discontinuous groups, the gradient sample groups appear to display more sharply increased local strain (Figures 6, 7).

\subsection{Local strain gradient maxima}

Maximum changes in local strain profiles were taken from individual line scans in order to obtain local strain gradient maxima for each group. Local strain gradients were significantly increased in composites containing arrays of discontinuous and gradient fibers compared to the continuous fiber arrays (Figure 8A). Significantly increased maximum local strains were observed in discontinuous and gradient fiber geometries compared to the continuous sample that was heightened in variants with $2 \mathrm{~mm}$ Amp sinusoidal fibers (Figure 8B). Given the need to create transitional specimens for future tendon-to-bone repair applications, we also examined the local strain maximum experienced within the middle third of the specimen alone to better 
quantify the differences between the discontinuous and gradient specimens. Not surprisingly, the maximum local strain experienced in the transitional zone was significantly increased in the discontinuous vs. continuous fiber geometries. Interestingly, incorporation of the gradient fiber transition resulted in significantly reduced local strain maxima compared to the discontinuous fiber array, identifying a path to potentially reduce local strain fields in regions of biomaterial transition (Figure 8C).

\section{4: Discussion}

Orthopedic tissue engineering often requires the ability to regenerate multiple tissues at once, using biomaterials which have tunable strength and toughness to match local mechanical properties of not just one, but multiple healthy tissues. However, high mechanical properties are often traded for porosity and high specific surface area, which better support cellular penetration, attachment, and nutrient biotransport. We have previously described a composite design, originally inspired by the longitudinal fibers found as reinforcing structures in plant stems (Speck and Burgert, 2011), as a potential solution to this trade-off, which incorporates ABS as a high strength fiber support within a highly porous collagen scaffold to create a composite with the best attributes of both (Mozdzen et al., 2016). Although successful as a model system, ABS is not an ideal polymer for long-term development of fiber-reinforced composites, which must allow incorporating of a wider range of functionality such as cell-adhesion and biodegradability in vivo.

Here we describe an approach to mechanically reinforce a collagen scaffold under development for tendon-to-bone junction (TBJ) repair applications. This work expands upon our previous 
work of the incorporation of ABS fibers arrays as a model polymer (Mozdzen et al., 2016) by replacing ABS with more biologically suitable and biodegradable poly-lactic acid (PLA), commonly used in biomaterial applications that is twice as stiff as ABS (Pu and Komvopoulos, 2014; Serra et al., 2013). While our previous work focused on spatially-homogenous fiber arrays, this work explores more complex fiber designs with regions containing distinct fiber geometries for the purpose of developing mechanically-graded composite biomaterial for multi-tissue regeneration. This work further explores not only the bulk mechanical properties of heterogeneous fiber arrays, but also local strain profiles to further define the design parameters required to tailor composite construct bulk mechanical properties. To facilitate the analysis of local strain fields via DIC, all mechanical tests for this project were performed in the nonhydrated (dry) state. We have previously shown that while hydrated CG scaffolds show reduced elastic moduli than non-hydrated scaffolds, the hydrated and dry scaffolds all show characteristic stress-strain behavior characteristic of low-density, open-cell foams (Caliari et al., 2015b; Caliari et al., 2014; Harley et al., 2007; Weisgerber et al., 2013a). Further, we have shown that composites formed from collagen scaffolds and polycaprolactone (PCL) support frame show identical mechanical performance in the dry and hydrated state due to the presence of the PCL phase (Weisgerber et al., 2016). While we do not expect significant short-term changes in the mechanical performance of the PLA-collagen composite due to the slow hydrolytic degradation of PLA, the ability to separately tailor the degradation characteristics of the CG scaffold via chemical crosslinking across a wide range (<2wks to $>2$ years) of half-lives (Harley et al., 2004) suggests future efforts to match PLA and collagen degradation rates with new tissue formation. Ongoing work is evaluating mechanical performance and cell response in PLA-collagen composites using in vitro culture in a tensile strain bioreactor. 
Homogenous biomaterials are relatively simple to characterize mechanically, but heterogeneous materials contain complex mechanical interactions, especially at the interface between discrete compartments (tendon, bone) or between distinct composite materials (CG,PLA), and require the correlation of local mechanical properties to properly describe the complete system. While the overall bulk mechanics performed as expected (elastic modulus: $E_{\text {straight }}>E_{1 \mathrm{~mm} \text { Amp }}>E_{2 \mathrm{~mm} \text { Amp }}$; elastic modulus and peak stress: $E_{\text {gradient }}>E_{\text {discontinuous }}>E_{\text {continuous; }}$; toughness: no change), local mechanical properties in our heterogeneous samples were more complex.

Although we observed higher elasticity, corresponding to reduced elastic modulus and higher strain at failure in the $2 \mathrm{~mm} \mathrm{Amp} \mathrm{sample} \mathrm{group} \mathrm{(Figure} \mathrm{2),} \mathrm{local} \mathrm{profiles} \mathrm{demonstrated}$ significantly higher strain gradients in discontinuous and gradient samples compared to continuous fiber geometries (Figures 8A). Comparatively, local strain maxima were significantly increased in the discontinuous and gradient fiber geometries with $2 \mathrm{~mm}$ amplitude when compared to the continuous sample, but were unchanged in the $1 \mathrm{~mm}$ amplitude fibers (Figures 8B). This is likely due to the mismatch in mechanical properties as the fibers transition from straight to sinusoidal, and this mismatch is more pronounced as the fiber has a larger amplitude $(2 \mathrm{~mm}>1 \mathrm{~mm})$. When engineering composite materials for increased strength in tissue engineering, the distribution of strain between composite materials remains an important design criterion, both due to concerns of failure and local cell mechanotransduction-based responses.

One of the challenges in the field of orthopedic tissue regeneration is to effectively dissipate stress concentrations between mechanically mismatched materials and move local regions of 
high strain gradients away from the interface between discrete compartments. In vivo, rotator cuff repairs often fail at the interface between tendon and bone due to these high stress concentrations (Genin et al., 2009). We have proposed a fiber reinforced collagen composite with a gradual transition (gradient) in fiber geometry from straight to sinusoidal to address this need. Not surprisingly, this composite effectively alters the local strain profiles across the scaffold in a manner different from those seen for a fiber design that contained a discontinuous geometry (straight-sinusoidal transition). Importantly, when the transitional region (middle 1/3) of the composite was examined specifically, gradient fiber arrays significantly decreased the local strain maxima experienced within the composite compared to the discontinuous fiber arrays (Figure 8C). This finding reinforces the idea that the manner in which the fiber transition takes place can significantly impact local mechanical behavior, making it a critical design parameter for future collage-PLA composites that contain both a scaffold transition and a transition in the fiber reinforcement. Not surprisingly, while the maximum local strain was decreased at the transitional region, overall strain gradients across discontinuous and gradient fiber geometries remained the same, suggesting that while strain maxima were shifted spatially, they were not eliminated. This result provides a cautionary insight into the cost of shifting the location of high strain gradients. Although the overall elastic modulus and peak stress are significantly higher in the fiber composite containing a gradual transition region (gradient), this is at the expense of high regions of local strain in the sinusoidal region.

Current studies in multi-tissue regeneration are exploring a wide range of design strategies to combine multiple materials into a single construct (Almodóvar et al., 2013; Atesok et al., 2014; Chen et al., 2015; Farnebo et al., 2014). However, the field of research exploring how these 
multi-material constructs behave on a local scale under mechanical load is extremely important for the dynamically active tissues found in the musculoskeletal system. The knowledge of local mechanical behavior is extremely vital as mechanically-sensitive cells are integrated into the biomaterial to ensure that proper loading conditions are met, but not exceeded (Chaudhuri et al., 2016; Foolen et al., 2014). Incorporating PLA fiber arrays within a collagen composite allows the ability to increase and tune bulk mechanical properties, but also highlights the need to carefully consider local strain fields in composite biomaterials that integrate multiple mechanically dissimilar materials into a single construct. This work clearly illustrates opportunities and challenges associated with complex composite design. We have demonstrated a trade-off in increased overall stiffness with allowing higher areas of local strain to form, as well as showing that shifting regions of high strain gradients from a discontinuity at the transition between fiber geometries (discontinuous v. gradient) allows larger strain gradients to form in a region of the sample further removed from the graded transition zone between fiber geometries.

\section{5: Conclusions}

The need to balance multiple requirements in the design of multi-compartment tissue engineering composites for musculoskeletal applications requires a new design toolbox. Herein we demonstrate a composite scaffold design that integrates more arrays of PLA fibers with tunable local mechanical properties into a CG scaffold to create a collagen-PLA composite with spatially tunable mechanical performance under applied tensile strain. Fiber reinforcement provides an avenue to significantly increase the bulk mechanical strength of the composite, but also the ability to spatially tailor the local mechanical performance of the composite via the use of 
spatially-variable fiber arrays. Together, this work further expands the toolbox of multi-tissue biomaterials by providing key insights into previously undemonstrated tradeoffs in bulk mechanical strength and local mechanical performance.

\section{Acknowledgements}

The authors would like to acknowledge assistance from Ryan Rodgers the creation of AutoDesk

fiber design files. Research reported in this publication was supported by the National Institute of Arthritis and Musculoskeletal and Skin Diseases of the National Institutes of Health under Award Numbers R21 AR063331. LCM was partially funded via National Science Foundation Grant 0965918 IGERT: Training the Next Generation of Researchers in Cellular \& Molecular Mechanics and BioNanotechnology, as well as the Support for Under-Represented Groups in Engineering (SURGE) Fellowship and the DuPont Science and Engineering Fellowship. This research was carried out in part in the Frederick Seitz Materials Research Laboratory Central Facilities, University of Illinois, which are partially supported by the U.S. Department of Energy under grants DE-FG02-07ER46453 and DE-FG02-07ER46471. 


\section{Figure Captions}

Figure 1. Fabrication of PLA fiber reinforced composites. A) PLA fiber reinforced composites are fabricated by incorporating an array of PLA fibers within the liquid collagen suspension prior to lyophilization. B) A library of collagen-PLA composites were created using PLA fiber arrays with continuous, discontinuous, or gradient profiles along their length that employed either $1 \mathrm{~mm}$ or $2 \mathrm{~mm}$ sinusoidal amplitudes.

Figure 2. Bulk mechanical characterization of PLA-collagen composites. A) Elastic modulus of composites containing uniform fiber arrays decreased significantly as the PLA fiber array sinusoidal amplitude increased. B) The bulk elastic modulus increased with increasing complexity of the spatiallygraded fiber arrays (Gradient $>$ Discontinuous $>$ Continuous) for both $1 \mathrm{~mm}$ and $2 \mathrm{~mm}$ fiber amplitudes. *: $\mathrm{p}<0.05$ vs. $2 \mathrm{~mm} \mathrm{Amp. \wedge}$ : $\mathrm{p}<0.05$ vs. Continuous sample with same amplitude.

Figure 3. Mechanical behavior at failure ( $2 \mathrm{~mm}$ amp samples). A) Representative images at the start of tensile testing and at the point of failure for the library of $2 \mathrm{~mm}$ sinusoidal amplitude fiber arrays. Failure occurred randomly in the continuous samples, but occurred in the sinusoidal region of the discontinuous and gradient transition samples. B) Toughness of the PLA-collagen composite remained constant for all groups. C) Peak stress was increased significantly in the gradient fiber array group. *: $p<$ 0.05 versus all other samples.

Figure 4. Strain fields within PLA-collagen composited under stain. Representative strain maps across the PLA-collagen composites under increasing levels of applied (bulk) strain $(0-3.7 \%)$. Composites containing discontinuous and gradient fiber arrays showed spatially variable stain fields, which correspond to the sinusoidal compartment of the fiber reinforcement. Composites containing $1 \mathrm{~mm}$ Amp fibers displayed sharper areas of local strain vs. those containing $2 \mathrm{~mm}$ Amp fiber arrays.

Figure 5. Digital image correlation and line scan analysis. Line scans were taken along the length of the specimen were used to quantify the strain distribution across each sample as well as calculate the maximum strain gradient experienced within each sample as a function of position.

Figure 6. Local stress concentrations with increasing bulk strain. Representative images of local strain fields within the PLA-collagen composite as a function of applied bulk strain depicted as a function of applied bulk strain $(0-3.7 \%)$. Composites containing arrays of continuous fiber geometries displayed local areas of high periodic strain more evenly spread than the $2 \mathrm{~mm} \mathrm{Amp}$ samples. Composites formed from arrays of discontinuous and gradient fiber arrays showed high areas of periodic strain localized to the region of the composite that incorporated sinusoidal fibers.

Figure 7. Distribution of local strain concentrations across the PLA-collagen composites (bulk strain: 2.3\%). Representative images of local strain fields within the PLA-collagen composite as a function of applied bulk strain, depicted as the mean and deviation of strain along the composite's length. Composites containing arrays of continuous fiber geometries displayed local areas of high periodic strain, more evenly spread in composites with $2 \mathrm{~mm}$ Amp fibers. Regions of elevated strain were in composites containing arrays of discontinuous and gradient fibers showed highest levels of strain localized to the sinusoidal fiber region within the composite.

Figure 8. Local strain gradient maxima within the fiber reinforced composites. A) Strain gradients were significantly increased in the discontinuous and gradient groups compared to the continuous fiber geometry. B) Local strain maxima observed throughout the entire composite as a function of applied bulk strain. C) Local strain maxima in the transitional zone (middle 1/3) of the composite were significantly 
impacted by the choice of fiber transition, with reduced local max strain in arrays with gradient vs.

discontinuous fiber geometries. *: $p<0.05$ versus samples with the same amplitude. ${ }^{\wedge}: p<0.05$ versus samples with the same compartment geometry but different amplitude. ${ }^{* *}: p<0.05$ versus discontinuous compartment geometry. 


\section{References}

Abd Elhady, A.A., 2010. Sress and strain concentration factors for plate with small notch subjected to biaxial loading - Three dimensional finite element analysis. Ain Shams Engineering Journal 1, 139-145.

Almodóvar, J., Crouzier, T., Selimović, Š., Boudou, T., Khademhosseini, A., Picart, C., 2013. Gradients of physical and biochemical cues on polyelectrolyte multilayer films generated via microfluidics. Lab on a Chip 13.

Atesok, K., Doral, M.N., Karlsson, J., Egol, K.A., Jazrawi, L.M., Coelho, P.G., Martinez, A., Matsumoto, T., Owens, B.D., Ochi, M., Hurwitz, S.R., Atala, A., Fu, F.H., Lu, H.H., Rodeo, S.A., 2014. Multilayer scaffolds in orthopaedic tissue engineering. Knee Surg Sports Traumatol Arthrosc, 1-9.

Caliari, S., Harley, B., 2014. Collagen scaffolds incorporating coincident gradations of structural and biochemical instructive cues for osteotendinous junction engineering,. In Preparation.

Caliari, S.R., Grier, W.K., Weisgerber, D.W., Mahmassani, Z., Boppart, M.D., Harley, B.A.C., 2015a. Collagen scaffolds incorporating coincident gradations of instructive structural and biochemical cues for osteotendinous junction engineering. Advanced healthcare materials 4, 831837.

Caliari, S.R., Grier, W.K., Weisgerber, D.W., Mahmassani, Z., Boppart, M.D., Harley, B.A.C., 2015b. Collagen scaffolds incorporating coincident patterns of instructive structural and biochemical cues for osteotendinous junction engineering. Adv Healthc Mater 4, 831-837.

Caliari, S.R., Mozdzen, L.C., Armitage, O., Oyen, M.L., Harley, B.A.C., 2014. Periodically perforated core-shell collagen biomaterials balance cell infiltration, bioactivity, and mechanical properties. J Biomed Mater Res A 102, 917-927.

Caliari, S.R., Ramirez, M.A., Harley, B.A.C., 2011. The development of collagen-GAG scaffoldmembrane composites for tendon tissue engineering. Biomaterials 32, 8990-8998.

Chandrashekar, N., Slauterbeck, J., Hashemi, J., 2012. Effects of cyclic loading on the tensile properties of human patellar tendon. The Knee 19, 65-68.

Chaudhuri, O., Gu, L., Klumpers, D., Darnell, M., Bencherif, S.A., Weaver, J.C., Huebsch, N., Lee, H.-P., Lippens, E., Duda, G.N., Mooney, D.J., 2016. Hydrogels with tunable stress relaxation regulate stem cell fate and activity. Nature Materials 15, 326-334.

Chen, S., Nakamoto, T., Kawazoe, N., Chen, G., 2015. Engineering multi-layered skeletal muscle tissue by using 3D microgrooved collagen scaffolds. Biomaterials 73, 23-31.

Farnebo, S., Woon, C.Y., Kim, M., Pham, H., Chang, J., 2014. Reconstruction of the TendonBone Insertion With Decellularized Tendon-Bone Composite Grafts: Comparison With Conventional Repair. The Journal of Hand Surgery 39, 65-74.

Ferreira, A.M., Gentile, P., Chiono, V., Ciardelli, G., 2012. Collagen for bone tissue regeneration. Acta Biomaterialia 8, 3191-3200.

Foolen, J., Janssen-van den Broek, M.W.J.T., Baaijens, F.P.T., 2014. Synergy between Rho signaling and matrix density in cyclic stretch-induced stress fiber organization. Acta Biomaterialia 10, 1876-1885. 
Galatz, L.M., Sandell, L.J., Rothermich, S.Y., Das, R., Mastny, A., Havlioglu, N., Silva, M.J., Thomopoulos, S., 2006. Characteristics of the rat supraspinatus tendon during tendon-to-bone healing after acute injury. Journal of Orthopaedic Research 24, 541-550.

Genin, G.M., Kent, A., Birman, V., Wopenka, B., Pasteris, J.D., Marquez, P.J., Thomopoulos, S., 2009. Functional Grading of Mineral and Collagen in the Attachment of Tendon to Bone. Biophysical Journal 97, 976-985.

Harley, B.A., Leung, J.H., Silva, E.C., Gibson, L.J., 2007. Mechanical characterization of collagen-glycosaminoglycan scaffolds. Acta Biomater 3, 463-474.

Harley, B.A., Lynn, A.K., Wissner-Gross, Z., Bonfield, W., Yannas, I.V., Gibson, L.J., 2010. Design of a multiphase osteochondral scaffold III: fabrication of layered scaffolds with continuous interfaces. J Biomed Mater Res A 92, 1078-1093.

Harley, B.A., Spilker, M.H., Wu, J.W., Asano, K., Hsu, H.P., Spector, M., Yannas, I.V., 2004. Optimal degradation rate for collagen chambers used for regeneration of peripheral nerves over long gaps. Cells Tissues Organs 176, 153-165.

Jones, E., 2013. Improved Digital Image Correlation (DIC).

Kanungo, B.P., Silva, E., Vliet, K.V., Gibson, L.J., 2008. Characterization of mineralized collagen-glycosaminoglycan scaffolds for bone regeneration. Acta Biomaterialia 4, 490-503.

Killian, M.L., Cavinatto, L., Galatz, L.M., Thomopoulos, S., 2012. Recent advances in shoulder research. Arthritis Research \& Therapy 14.

Levingstone, T.J., Matsiko, A., Dickson, G.R., O’Brien, F.J., Gleeson, J.P., 2014. A biomimetic multi-layered collagen-based scaffold for osteochondral repair. Acta Biomaterialia 10, 19962004.

Li, X., Xie, J., Lipner, J., Yuan, X., Thomopoulos, S., Xia, Y., 2009. Nanofiber scaffolds with gradations in mineral content for mimicking the tendon-to-bone insertion site. Nano Letters 9, 2763-2768.

Lionello, G., Sirieix, C., Baleani, M., 2014. An effective procedure to create a speckle pattern on biological soft tissue for digital image correlation measurements. Journal of the Mechanical Behavior of Biomedical Materials 39, 1-8.

Lipner, J., Liu, W., Liu, Y., Boyle, J., Genin, G.M., Xia, Y., Thomopoulos, S., 2014. The mechanics of PLGA nanofiber scaffolds with biomimetic gradients in mineral for tendon-to-bone repair. Journal of the Mechanical Behavior of Biomedical Materials 40, 59-68.

Maganaris, C.N., Paul, J.P., 1999. In vivo human tendon mechanical properties. J Physiol 521, 307-313.

Marino, M., Vairo, G., 2014. Stress and strain localization in stretched collagenous tissues via a multiscale modelling approach. Comput Methods Biomech Biomed Engin 17, 11-30.

Mathew, A.P., Oksman, K., Pierron, D., Harmand, M.-F., 2012. Biocompatible Fibrous Networks of Cellulose Nanofibres and Collagen Crosslinked Using Genipin: Potential as Artificial Ligament/Tendons. Macromolecular Bioscience, n/a-n/a.

Mozdzen, L.C., Rodgers, R., Banks, J.M., Bailey, R.C., Harley, B.A.C., 2016. Increasing the strength and bioactivity of collagen scaffolds using customizable arrays of 3D-printed polymer fibers. Acta Biomater 15, 25-33.

Myers, K.M., Socrate, S., Paskaleva, A., House, M., 2010. A study of the anisotropy and tension/compression behavior of human cervical tissue. J Biomech Eng 132, 021003. 
O’Brien, F.J., Harley, B.A., Yannas, I.V., Gibson, L., 2004. Influence of freezing rate on pore structure in freeze-dried collagen-GAG scaffolds. Biomaterials 25, 1077-1086.

$\mathrm{Pu}$, J., Komvopoulos, K., 2014. Mechanical properties of electrospun bilayer fibrous membranes as potential scaffolds for tissue engineering. Acta Biomaterialia 10, 2718-2726.

Qu, J., Thoreson, A.R., Chen, Q., An, K.-N., Amadio, P.C., Zhao, C., 2013. Tendon gradient mineralization for tendon to bone interface integration. Journal of Orthopaedic Research, n/a-n/a.

Sano, H., Hatta, T., Yamamoto, N., Itoi, E., 2013. Stress Distribution Within Rotator Cuff Tendons With a Crescent-Shaped and an L-Shaped Tear. Am J Sports Med 41, 2262-2269.

Serra, T., Planell, J.A., Navarro, M., 2013. High-resolution PLA-based composite scaffolds via 3-D printing technology. Acta Biomaterialia 9, 5521-5530.

Smith, L.J., Deymier, A.C., Boyle, J.J., Li, Z., Linderman, S.W., Pasteris, J.D., Xia, Y., Genin, G.M., Thomopoulos, S., 2016. Tunability of collagen matrix mechanical properties via multiple modes of mineralization. Interface Focus 6, 20150070.

Speck, T., Burgert, I., 2011. Plant stems: functional design and mechanics. Ann Rev Mater Res 41, 169-193.

Stops, A.J., McMahon, L.A., O'Mahoney, D., Prendergast, P.J., McHugh, P.E., 2008. A finite element prediction of strain on cells in a highly porous collagen-glycosaminoglycan scaffold. J Biomech Eng 130, 061001 (061011 pages).

Szabo, B., Babuska, I., 1991. Finite Element Analysis. John Wiley and Sons, New York.

Thomopoulos, S., Williams, G.R., Gimbel, J.A., Favata, M., Soslowsky, L.J., 2003. Variation of biomechanical, structural, and compositional properties along the tendon to bone insertion site. J Orthop Res 21, 413-419.

Weisgerber, D.W., Caliari, S.R., Harley, B.A.C., 2015. Mineralized collagen scaffolds induce hMSC osteogenesis and matrix remodeling. Biomater Sci 3, 533-542.

Weisgerber, D.W., Erning, K., Flanagan, C., Hollister, S.J., Harley, B.A.C., 2016. Evaluation of multi-scale mineralized collagen-polycaprolactone composites for bone tissue engineering. J Mech Behav Biomed Mater 61, 318-327.

Weisgerber, D.W., Kelkhoff, D.O., Caliari, S.R., Harley, B.A.C., 2013a. The impact of discrete compartments of a multi-compartment collagen-GAG scaffold on overall construct biophysical properties. J Mech Behav Biomed Mater 28, 26-36.

Weisgerber, D.W., Kelkhoff, D.O., Caliari, S.R., Harley, B.A.C., 2013b. The impact of discrete compartments of a multi-compartment collagen-GAG scaffold on overall construct biophysical properties. Journal of the Mechanical Behavior of Biomedical Materials 28, 26-36.

Yamaguchi, K., Ditsios, K., Middleton, W.D., Hildebolt, C.F., Galatz, L.M., Teefey, S.A., 2006. The Demographic and Morphological Features of Rotator Cuff DiseaseA Comparison of Asymptomatic and Symptomatic Shoulders. The Journal of Bone \& Joint Surgery 88, 16991704.

Yannas, I.V., Lee, E., Orgill, D.P., Skrabut, E.M., Murphy, G.F., 1989. Synthesis and characterization of a model extracellular matrix that induces partial regeneration of adult mammalian skin. Proc Nat Acad Sci 86, 933-937.

Zienkiewicz, O.C., 1977. The Finite Element Method, 3rd ed. McGraw-Hill, New York. 

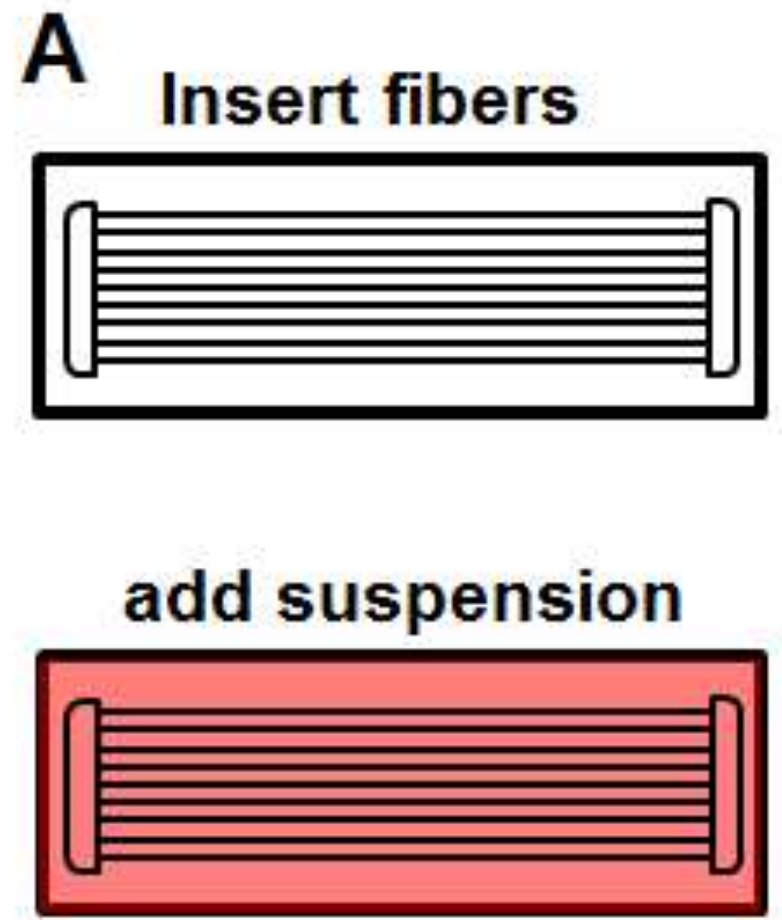

freeze-dry

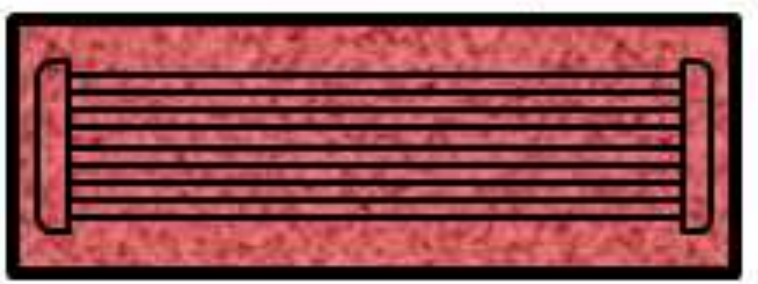

B

Continuous

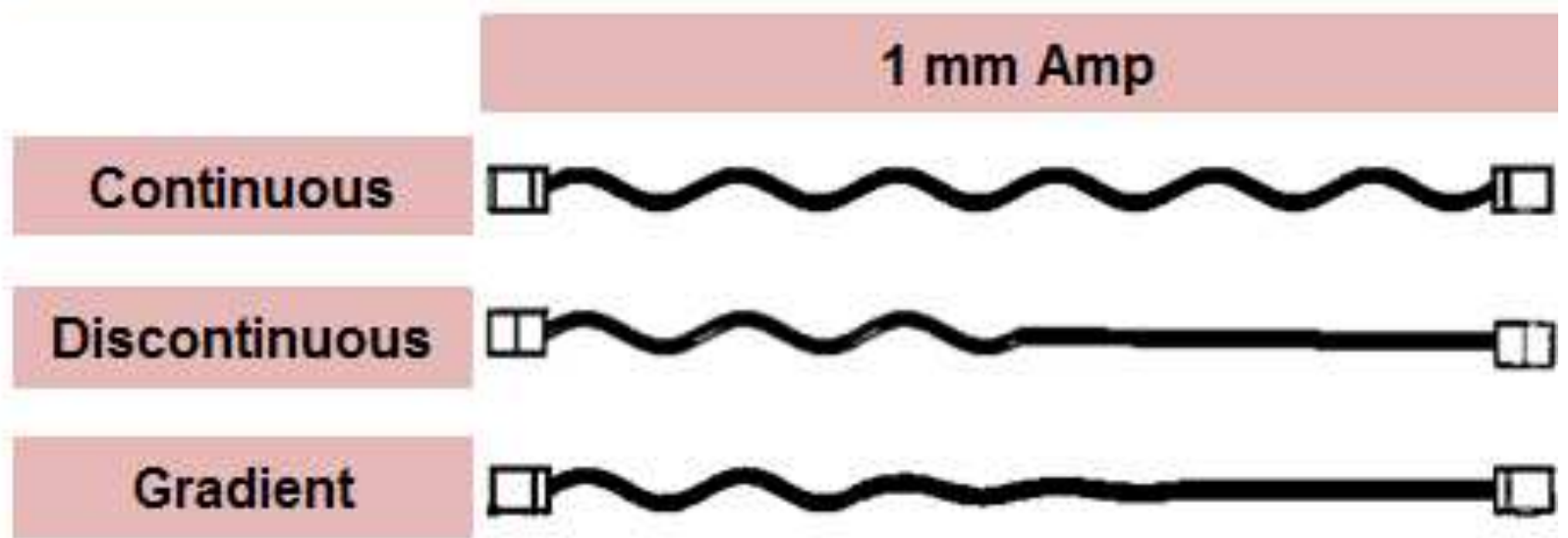

$2 \mathrm{~mm} \mathrm{Amp}$

Continuous

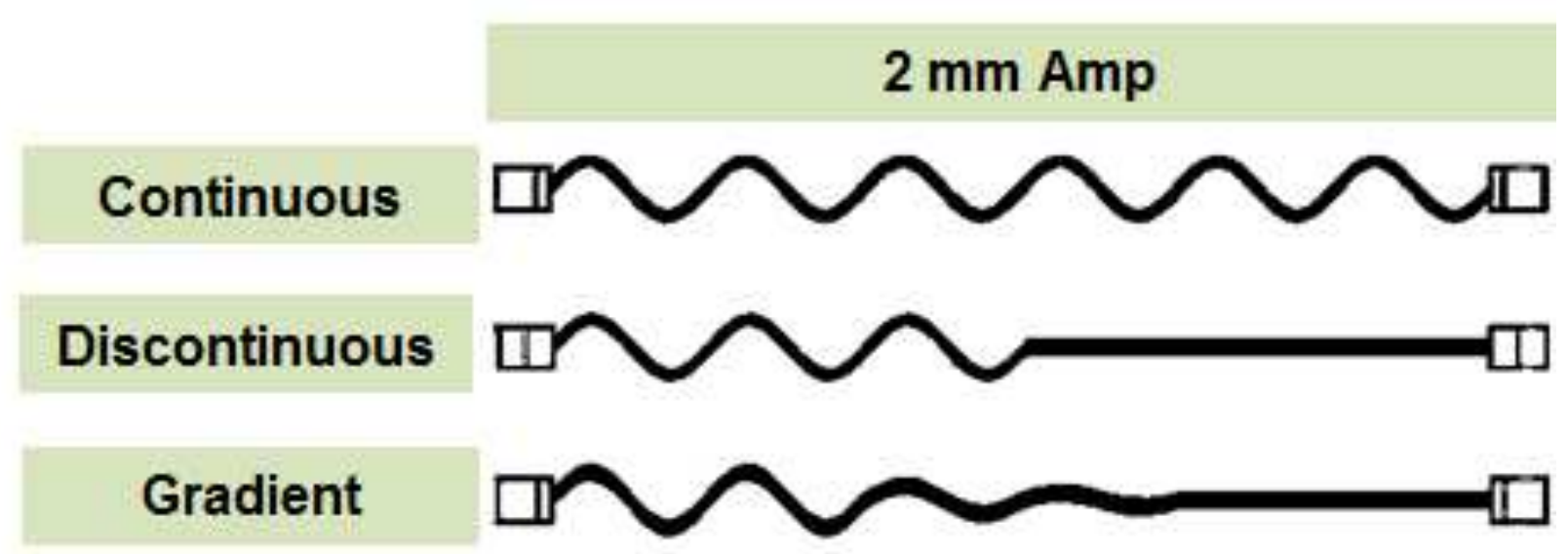

Discontinuous

Gradient

Straight पा

\section{1}



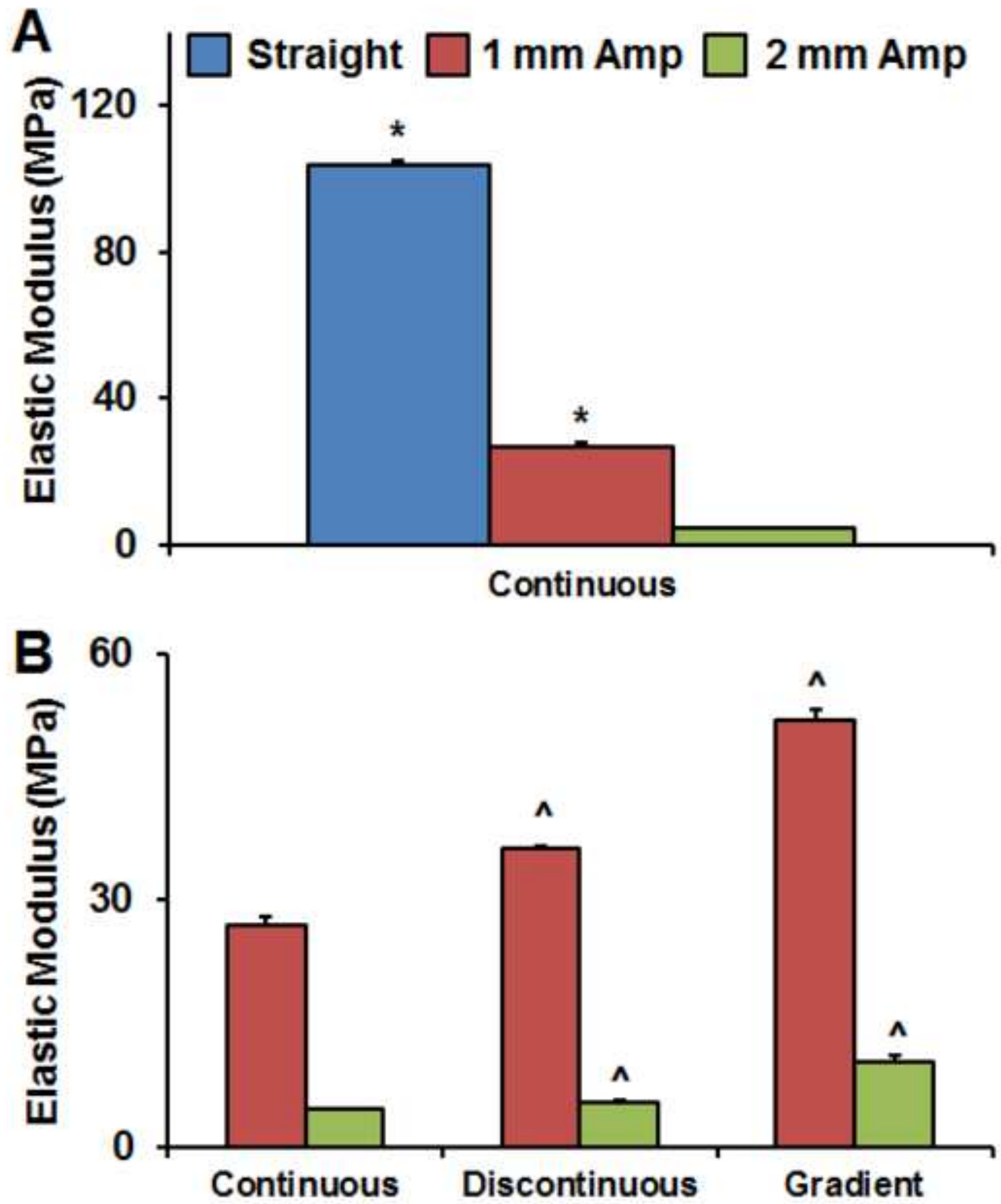

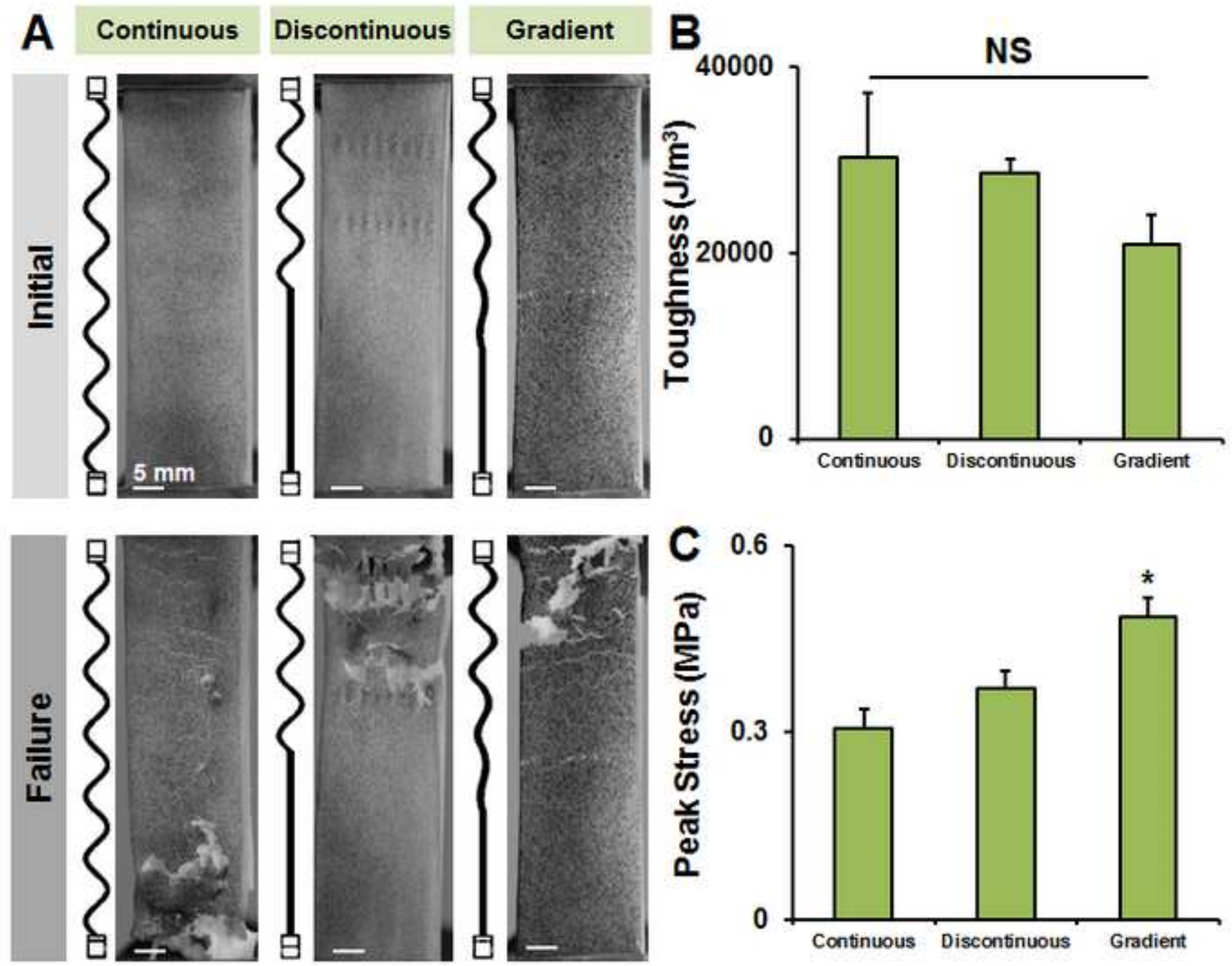


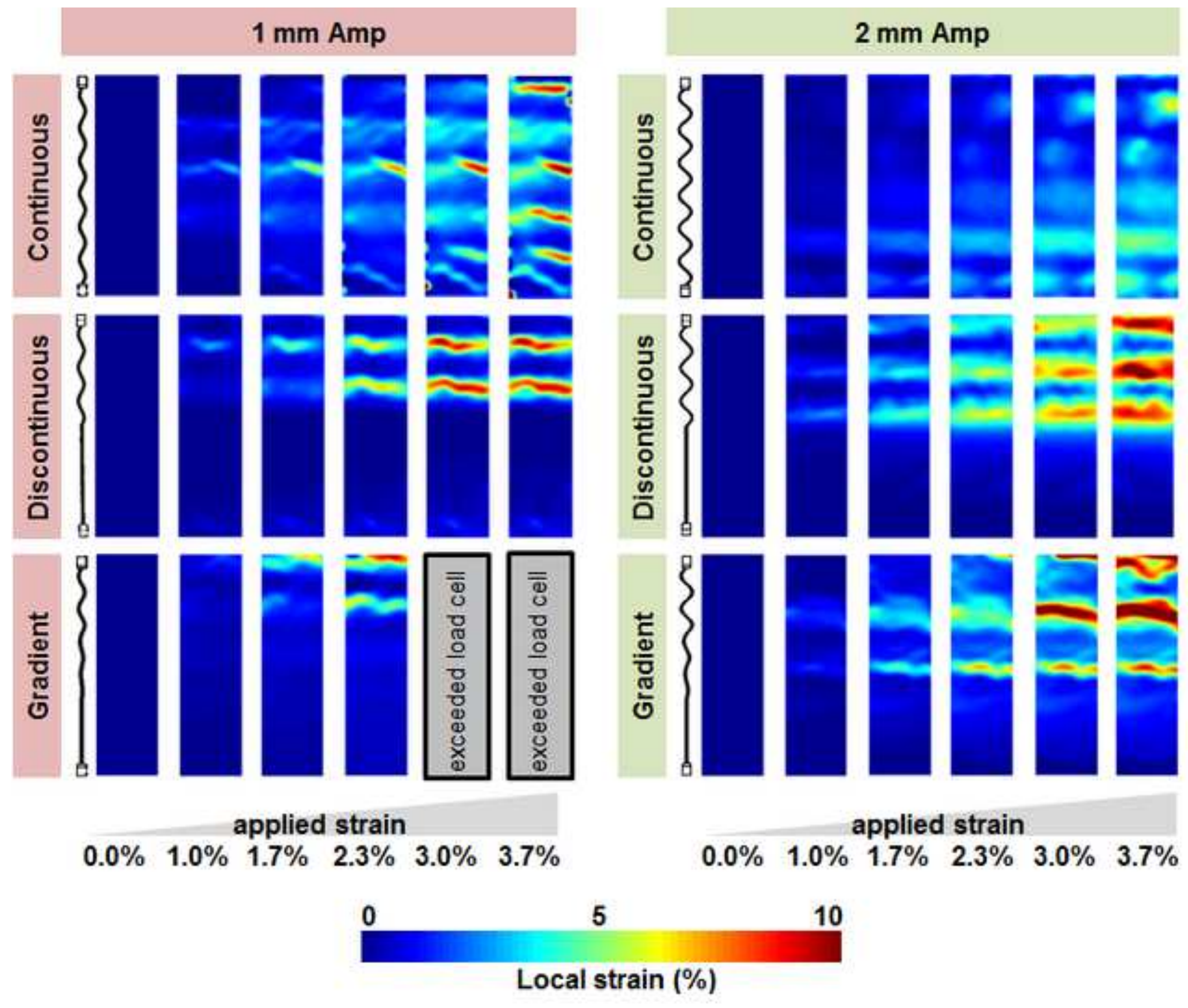




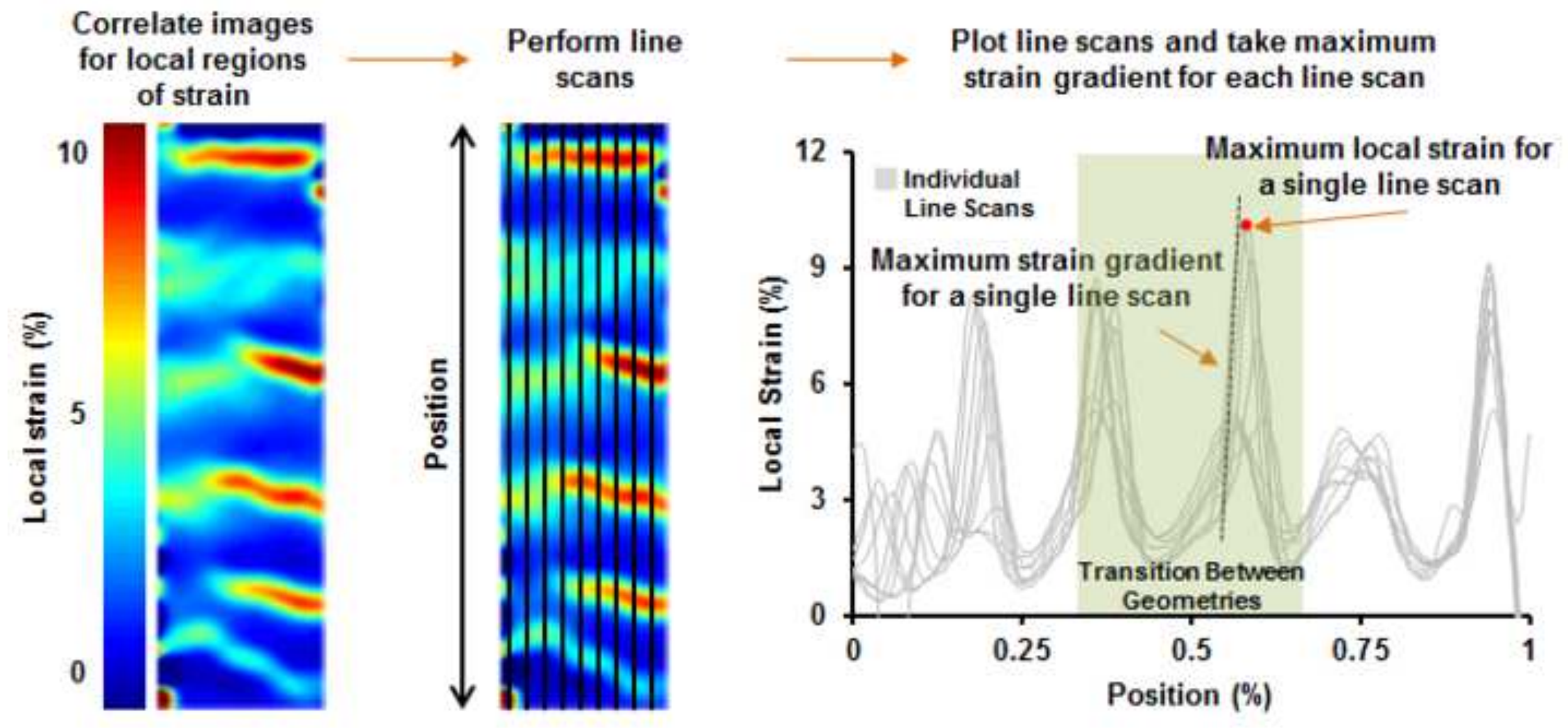



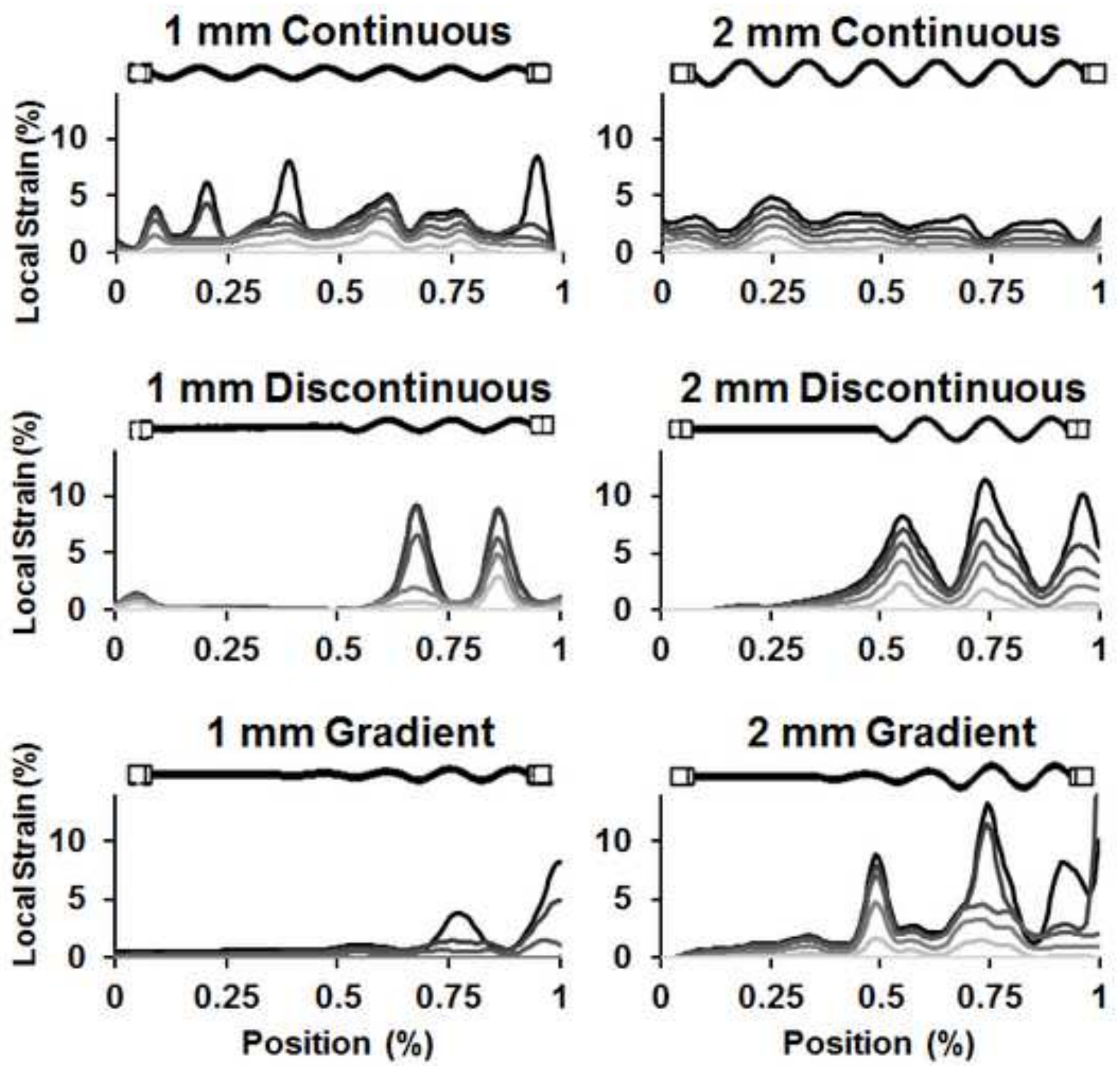

$\square 0.0 \% \quad \square 1.0 \% \quad \square 1.7 \%$

$2.3 \% \square 3.0 \% \square 3.7 \%$



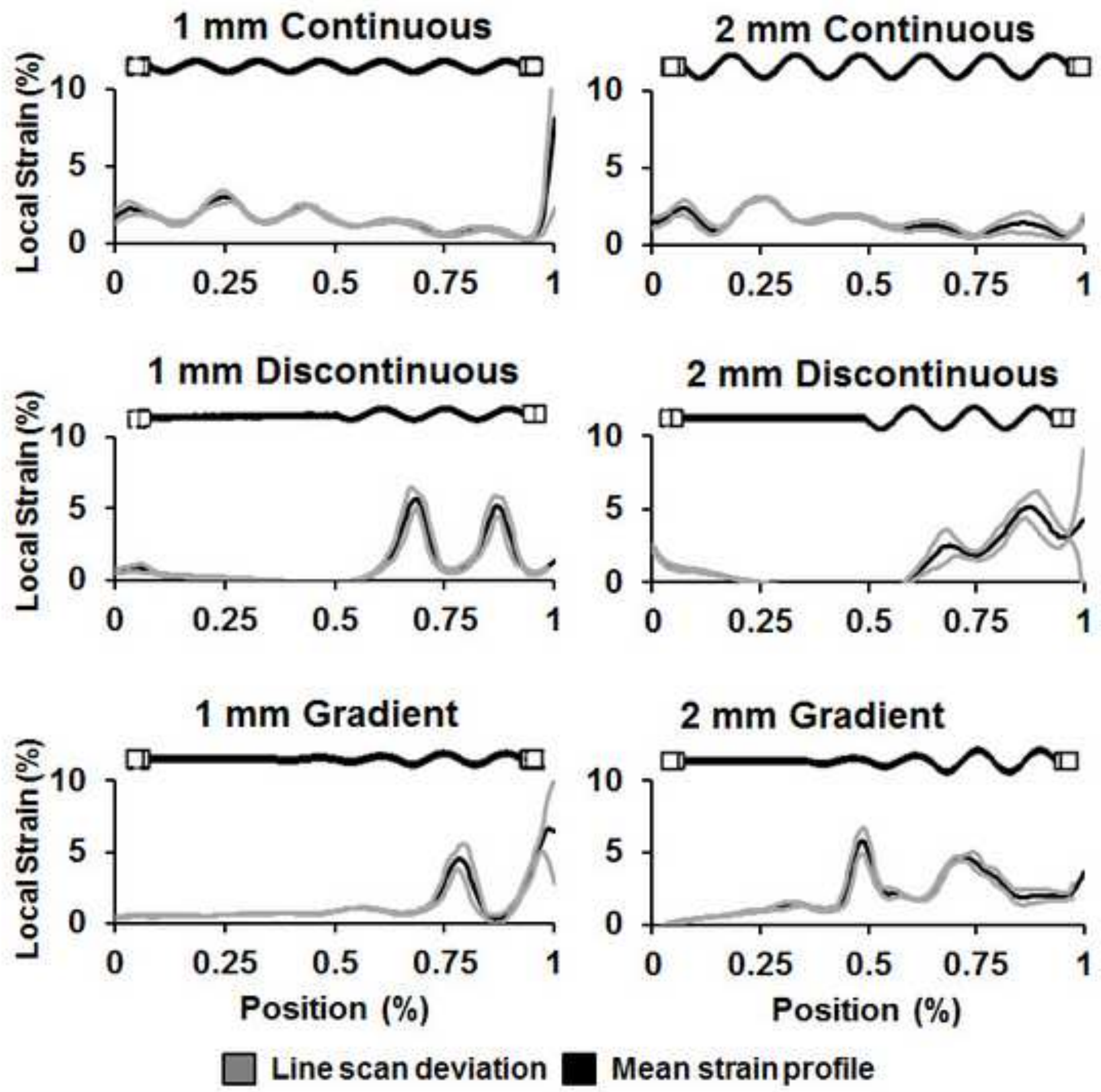

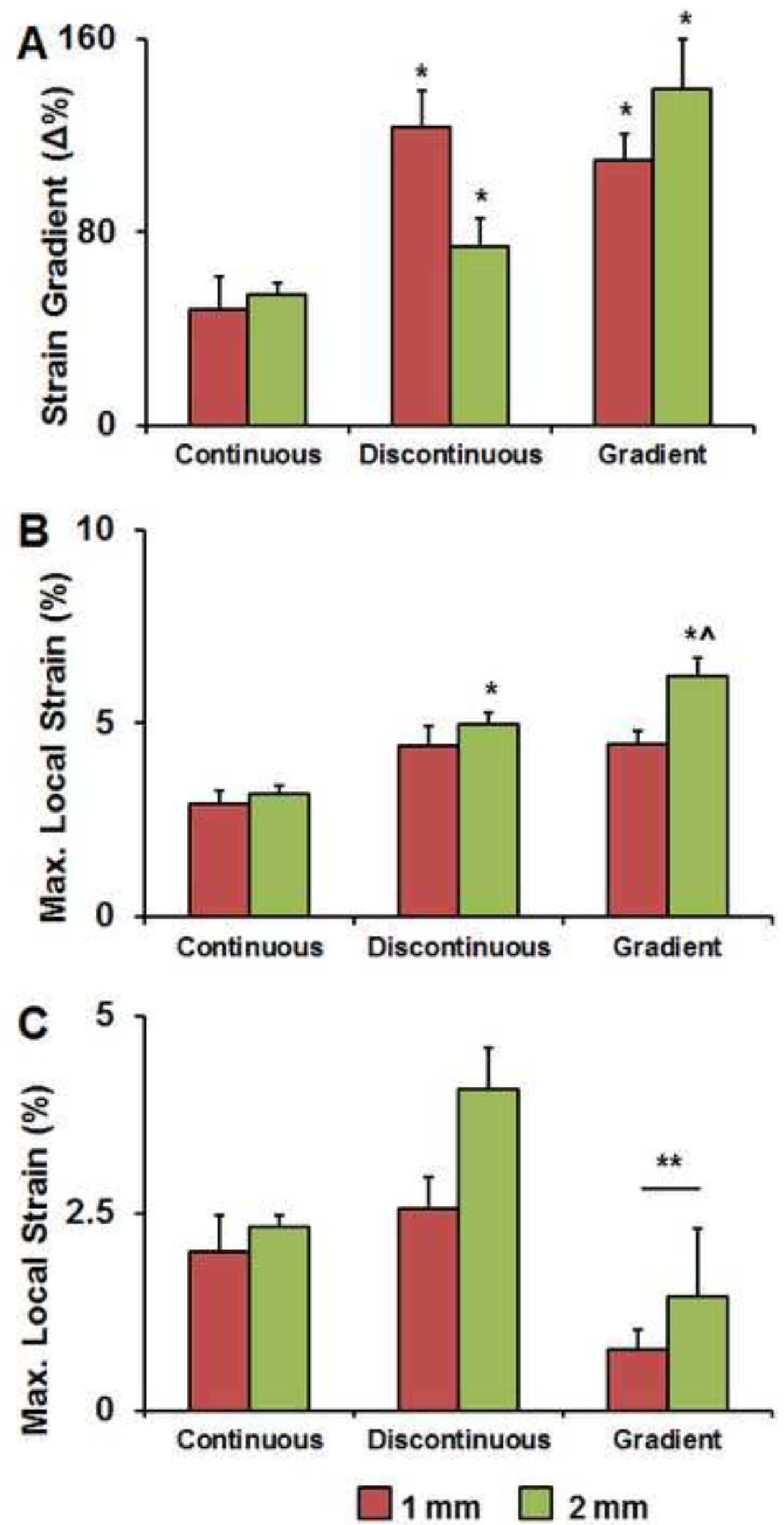